\title{
Human Amniotic Fluid Stem Cells as an Attractive Tool for Clinical Applications
}

\author{
Ourania Trohatou $^{1,2}$, Nicholas P. Anagnou ${ }^{1,2}$ and Maria G. Roubelakis*,1,2 \\ ${ }^{1}$ Laboratory of Biology, University of Athens, School of Medicine, Athens, Greece; ${ }^{2}$ Cell and Gene Therapy Laboratory, \\ Centre of Basic Research II, Biomedical Research Foundation of the Academy of Athens (BRFAA), Athens, Greece
}

\begin{abstract}
Abstrac: Recent studies support cell based therapies for several diseases. Human fetal stem cells have received much attention for developing new therapeutic strategies. Recently, our group and others have successfully isolated and expanded karyotypically normal stem cells from an alternative fetal source, the human second trimester amniotic fluid (AF) and performed a systematic phenotypic and molecular analysis. The main characteristics of amniotic fluid stem cells (hAFSCs) are their fetal origin, the high number of isolated cells, their wide differentiation properties and their rapid expansion in vitro. These characteristics render hAFSCs as a very attractive tool for clinical applications based on cell therapy. The use of hAFSC transplantation has been studied in a variety of disease animal models related to bone regeneration, myocardial infarction, acute kidney injury, acute hepatic failure, skin injury, ischemic hind limb or cancer. The major aim of this review is to summarize the advent of hAFSCs capabilities into novel therapeutic modalities and discuss their potential use in future pre-clinical and clinical studies.
\end{abstract}

Keywords: Amniotic fluid, animal models, cell transplantation, c-Kit, fetal stem cells, MSCs, stem cell therap.y.

\section{INTRODUCTION}

Regenerative medicine introduces methods to replace or regenerate cells, tissue or organs in order to restore and establish normal function [1]. The three strategies used in regenerative medicine include cell based therapy, biomaterials and scaffolds seeded with cells. In all cases, autologous or allogeneic mature functional cells (cell replacement), modified human cells (cell based gene therapy) or stem cells have been used in transplantation [2].

There are several parameters to take under consideration for a successful cell based therapy. Basic tools for cell transplantation are the scaffolds, usually made by natural or synthetic materials with diverse bioactivity and mechanical strength. Scaffolds provide the appropriate environment for cellular growth and organogenesis. Recent studies have been focused on various applications based on stem cell transplantation seeded into scaffolds [3]. The type of stem cells together with the selection of the appropriate biomaterial remain a critical issue in cell based therapy. Embryonic stem cells (ESCs) have great potential due to their unique characteristics, however their use is limited by ethical considerations [4]. In addition, mesenchymal stem cells derived from the bone marrow (BM-MSCs) have been used with remarkable results in animal studies and clinical trials but also revealed several limitations. More recently, research has been

\footnotetext{
*Address correspondence to this author at the Laboratory of Biology, Medical School, University of Athens, Athens 115 27, Greece; Cell and Gene Therapy Laboratory Centre of Basic Research II Biomedical Research Foundation of the Academy of Athens (BRFAA), Athens 115 27, Greece; Tel. +30-210-6597-013; Fax +30-210-6597-545;

E-mail: mroubelaki@bioacademy.gr
}

focused on stem cells derived from fetal sources. Suchan appealing source used in transplantation studies is the amniotic fluid (AF) representing a novel and very promising alternative.

The cell therapy field has progressed to the forth therapy method of global healthcare, among therapies based on pharmaceuticals, biological manipulations and medical devises, covering billion dollars per year during the last decade [5]. A successful stem cell therapy approach requires a thorough study of the stem cell biology, the availability of well established animal models and the development of gene transfer methodologies to stem cells Fig. (1).

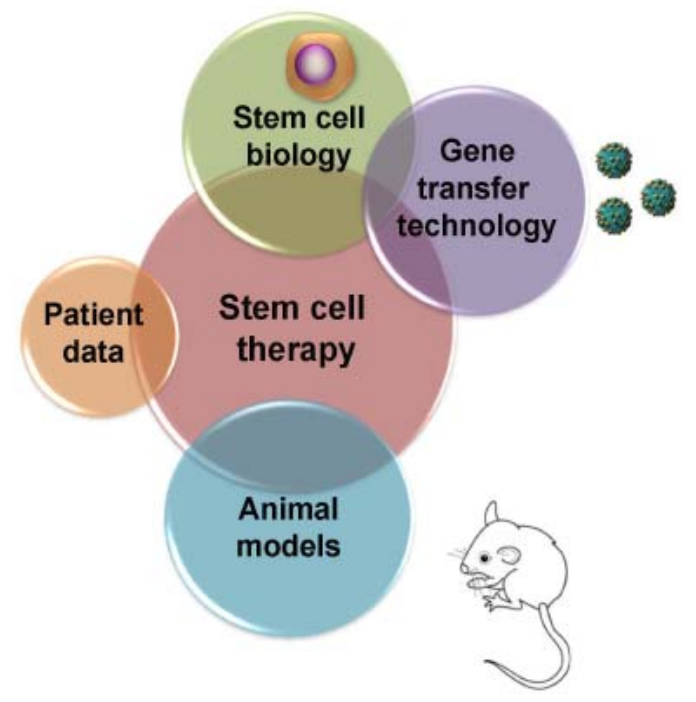

Fig. (1). Main aspects of stem cell therapy approach. 


\section{Amniotic Fluid Stem Cells}

The presence of stem cells in human amniotic fluid was firstly described in 2002 by Prusa et al. [6]. hAFSCs can be easily obtained from a small volume of second trimester AF, collected during routine amniocenteses [6-12] for prenatal diagnosis, a procedure with spontaneous abortion rate ranging from 0.06 to $0.5 \%[7,13,14]$. hAFSCs represent a heterogenous population characterized by a multipotent phenotype, high proliferation potential as well as wide differentiation properties $[7-12,15,16]$.

It was reported that hAFSC culture contains a small population of cells expressing the surface antigen c-Kit (CD117), the type III tyrosine kinase receptor of the stem cell factor [11]. Immunoselection of c-Kit positive hAFSCs can be performed by magnetic beads, and the isolated cells can be clonally expanded in culture [11].

Another type of hAFSCs is the amniotic fluid mesenchymal stem cells (hAF- MSCs) that can be easily obtained in culture with estimated frequency in the AF sample between $0.9 \%$ to $1.5 \%$ [7]. Recently distinct populations of hAF-MSCs were identified, with different proliferation and differentiation potential, termed according to their morphology, as spindle shaped (SS) and round shaped (RS) [12]. Mechanical isolation of both AF-MSC types was conducted, and revealed the RS cells as the most abundant population in the tested samples. However, $6 \%$ of the samples were enriched in the SS cell population which can expanded up to 30-50 passages with a normal karyotype and without showing any tumogenic potential after expansion in culture [7, 12].

\section{Amniotic Fluid Stem Cell as Therapeutic Tool}

During the last decade there is a strong ethical debate primarily concerning the destruction of human embryos for the isolation of hESCs. However, other stem cell types such as adult stem cells, fetal stem cells and induced pluripotent stem cells (iPS) overcome the ESC controversy. Such an example, of fetal stem cell population is the hAFSCs. About $90 \%$ of hAFSCs express the transcription factor Oct4, known to be expressed in pluripotent human stem cells, suggesting that hAFSCs belong to an intermediate developmental stage between embryonic and adult stem cells [11, 17]. $\mathrm{AF}$ is collected during scheduled amniocenteses through routine prenatal diagnosis without any ethical concerns [6-9]. In comparison, the isolation of hMSCs derived from an adult source such as bone marrow is a highly invasive and painful procedure and additionally, the number, the proliferation and the differentiation capacity of these cells decline with increasing age $[18,19]$.

\section{Immunological Properties}

In cell based therapies, an important limitation is the possibility of cell rejection. For this reason, hAFSCs represent an advantageous cell type for allogeneic transplantation as they have an inherently low immunogenic profile exhibiting low expression of HLA-ABC and no HLA-DR antigens [7, $8,11,12]$. Therefore, the use of hAFSCs reduces the risk of cell rejection and the potential of graft versus-host disease.

\section{Role in Anti-inflammatory Response}

Much of excitement surrounds the ability of hAFSCs to suppress inflammatory responses. c-Kit positive hAFSCs have been shown to inhibit the activation of lymphocytes in a dose dependent manner involving both direct and indirect interaction between stem cells and their secreted factors with the immune cells [20]. hAFSCs released several cytokines mediating in inflammatory response such as growth related oncogene (GRO), monocyte chemotactic protein family (MCP) as well as interleukin-6 (IL-6) [20]. Additionally, in our studies, we analyzed the cytokines secreted in the conditioned medium derived from human SS-AF-MSCs [21]. It was observed that SS-AF-MSCs expressed a number of cytokines and growth factors such as transforming growth factor beta-1 (TGF- $\beta 1$ ) and granulocyte macrophage colony stimulating factor (GM-CSF), responsible for downregulating the systemic inflammation. Therefore, hAFSCs exhibit immunomodulatory potential resembling MSCs.

\section{Virus Mediated Gene Transfer}

The scenario of using hAFSCs for therapeutic applications requires these cells to be genetically modified in order to deliver systematically the desired therapeutic gene to the injured area. To this end, Grifani et al. showed that hAFSCs can be transduced with adenovirus vectors with high efficiency [22]. In addition, no alterations on cell phenotype and their differentiation properties were observed. In our studies, human SS-AF-MSCs were successfully transduced in a virus dose-depended manner with GFP third generation lentivirus [12]. No silencing effects were noticed over the multiple culture passaging, maintaining the GFP expression. Also, GFP transduced SS-AF-MSCs retained their in vitro differentiation potential. These findings provide a proof of principle for designing modified hAFSCs for therapeutic applications.

\section{Absence of Teratoma Formation in vivo}

A major concern in stem cell based therapy is the formation of teratomas in vivo. However, no tumor formation was observed even 3 months after transplantation of human SSAF-MSCs or c-Kit positive hAFSCs into immune-deficient mice $[11,12]$. In contrast, hESCs often form teratocarcinomas when transplanted in vivo, acquiring frequently chromosomal aberrations [23-25].

\section{Preclinical Studies}

\section{Animal Models}

Animal models are recognized as powerful tools in research associated with several diseases. Clinical testing and development of novel therapeutic schemes depend on the availability of well established animal models. The use of preclinical models provides new sights in tissue engineering and enables cell transplantation for further investigation on the repair and the replacement of damaged tissues or organs. Well established transplantation models are needed to test the ability of transplanted cells to ameliorate the disease symptoms and also examine potential side effects [26]. In several studies, AFSCs have been used for autologous transplantation in respective animal models. This review provides 
an overview on the use of human AFSCs in animal models resembling severe diseases.

\section{Bone Defects}

Bone defects are commonly caused by injury, congenital disorders or surgery for bone cancer. Treatment of extensive bone defects indicates autologous bone replacement or the use of bone enhancement materials. An alternative approach has been focused on engineering biological bone grafts by culturing cells within 3D scaffolds under appropriate conditions for bone formation.

For the first time, De Coppi and his group presented the formation of tissue engineered bone from printed constructs of osteogenically differentiated hAFSCs in immune-deficient mice [11]. c-Kit positive hAFSCs were placed into an alginate/collagen composite gel, creating scaffold/cell constructs that were incubated for one week under osteogenic conditions prior implantation. The implanted printed constructs exhibited high mineralized tissue after 8 weeks as examined by histological analyses. The production of hard tissue within the cell seeded scaffolds was observed 4-5 months post implantation, confirming in this way the formation of high density tissue engineered bone [11].

\section{Myocardial Infarction}

Myocardial infarction is characterized by reduced blood supply of the heart muscle, usually due to atherosclerosis of the coronary arteries. Recently, stem cell therapy has been considered effective for treating ischemic heart disease. Interestingly, it has been shown that hAF-MSCs express cardiac specific genes under appropriate culture conditions and are able to integrate into normal and ishaemic heart explants, where they can differentiate into cardiocyte-like cells [27].

Chiavegato et al. investigated for the first time the in vivo therapeutic effect of hAFSCs in a rat model of myocardial infarction [28]. Left anterior descending coronary artery in female Sprague-Dawley rats was identified and ligated with a 7-0 polypropylene suture. After $20 \mathrm{~min}$, immunomagnetic sorted hAFSCs were transplanted in ischemic heart of immune-competent or immuno-deficient animals in the periphery of the damaged area in three distinct sites. Despite all expectations, transplanted hAFSCs were acutely rejected, because of the recipient's immune response whereas, in some cases, hAFSCs gave rise to chondro-osteogenic masses in the heart [28].

In a recent publication, Delo et al. confirmed the formation of such masses in the ventricular wall of infracted rats [29]. These data suggested that the chondro-osteogenic masses were formed independently of the hAFSCs injection into the myocardium. In particular, masses were correlated with the infarction size and the fractional shortening [29].

\section{Sciatic Nerve Injury}

The first evidence that hAFSCs have been able to survive and migrate after transplantation into the striatum of normal and ischemic rats was presented in 2007 by Cipriani et al. [30]. Notably, grafted cells migrated in several brain regions in normal animals and also intended to move to the injured regions. Based on these important findings many efforts have been focused on hAFSCs as a promising treatment on nerve injuries.

The peripheral nerve regeneration using hAF-MSCs was studied for the first time by Pan et al. [31]. A sciatic nerve injury was generated using Sprague Dawley rats. The left sciatic nerve was exposed under microscope examination and pressure was applied using a vessel clamp. The sciatic nerve crush effected nerve function, disrupted nerve integrity and provoked inflammatory response [31]. hAF-MSCs were embedded in fibrin glue containing the woven surgical and transplanted into the injured site immediately after crush. The rejection of cell transplantation was prevented with the use of cyclosporine. Granulocyte-colony stimulating factor (G-CSF) was also intraperitoneal administrated. This combined treatment showed increased nerve myelination and a significant improvement in motor function. G-CSF that was also expressed in hAF-MSCs, prevented the anti-apoptotic effect on implanted hAF-MSCs and in the same time had anti-imflammatory role [31].

In another study, the same group reported the beneficial effect of hyperbaric oxygen (HBO) on transplanted hAFMSCs in peripheral nerve regeneration [32]. The sciatic nerve injury model was generated as mentioned before and the transplantation of hAF-MSCs was performed using the same protocols. The rats received $\mathrm{HBO}$ after transplantation for seven days. HBO has been shown to inhibit proinflammatory cytokines synthesis leading to an antiinflammatory effect. The peripheral nerve regeneration observed may be related to the suppression of apoptosis in implanted hAF-MSCs and in the reduction of the inflammatory response [32].

Using as a backbone the same sciatic nerve injury model and the implantation method of hAFSCs, Pan et al. also investigated the combined therapeutic effect of fermented soybean extracts, termed as Natto [33]. Administration of Natto is proposed to rescue hAFSCs and Schwann cells from apoptosis resulting to neurological improvement.

The sciatic nerve crush model was also studied by Cheng et al. [34]. In particular, hAF-MSCs were modified to express human glia cell line-derived meurotrophic factor (GDNF) using adenoviral vector. GDNF-AF-MSCs were labeled with lipophilic fluorescence marker and then embedded in matrigel and transplanted to the injured nerve. To avoid rejection, cyclosporine was also used in this study. Rats treated with GDNF-AF-MSCs showed improvement in motor function and early regeneration of neurofilaments, as well as increased proliferation rate of Schwann cells in the distal end of the nerve. Simultaneously, nerve regeneration in the later phase was observed through increased myelination, vascular organization and decreased number of vascuoles [34].

A later publication came in 2012, regarding the sciatic nerve injury model by Yang et al. [35]. hAF-MSCs were intravenously administered according to their stromal cellderived factor- $1 \alpha$ (SDF-1 $\alpha)$ expression immediately or 7 days after injury. SDF- $1 \alpha$ was overexpressed in nerve and muscle after injury together with the chemokine receptor type-4 (CXCR-4). More importantly, SDF-1 $\alpha$ increased the 
migration and the wound healing properties of hAF-MSCs, supporting their engraftment in muscle and nerve. Transplanted cells were found in significant amounts in spleen, muscle and nerve, when administrated 7 days after the injury. In this study, neurobehavior improvement was observed based on the sciatic function index, the electrophysiology test and the computerized gait analysis. hAF-MSCs treatment showed regeneration of nerve and muscle tissue function by an increase in the number of axons, together with nerve myelination and high expression of neurotrophic factors and acetylcholine receptor. These findings suggested a nerve regeneration scenario focused on hAF-MSCs for peripheral nerve disorder [35].

\section{Acute Kidney Injury}

Acute tubular cell injury and renal dysfunction causes acute tubular necrosis. In 2007, Perin et al. reported renal differentiation of c-Kit positive AFS cells [36] and three years later showed the therapeutic potential of these cells in a mouse model for acute tubular necrosis [37]. The mouse model was generated by intramuscular injection with $50 \%$ hypertonic glycerol solution in water, following 22 hours deprivation of water. hAFSCs were labeled with CM-Dil and then carefully injected into the renal cortex of both kidneys. Evidence showed that c-Kit positive hAFSCs were present in the kidney for 21 days after transplantation and were differentiated into cells expressing adult proximal and distal tubular agglutinins. The levels of serum creatinine and blood urea nitrogen together with a decreased number of damaged tubules suggested that hAFSCs improved acute tubular injury. In addition, hAFSCs were observed to modulate the immune response expressing anti-inflammatory cytokines [37].

Another study by Hauser et al. also demonstrated that hAFSCs induce the recovery of murine experimental acute kidney injury [38]. The SCID acute kidney injury mice model was generated by intramuscular injection of glycerol, exposing the kidney to myoglobin and iron, creating in this way tubular injury. Transplanted hAFSCs were detected within the peritubular capillaries localized into the interstitium, but they did not integrate into the renal tissue. The intravenous injection of hAFSCs improved the renal function, enhanced tubular cell proliferation and reduced apoptosis. Also, the produced cytokines and growth factors in the conditioned medium of hAFSCs, suggested a combined mechanism for their therapeutic properties in acute kidney injury. More specifically, LIF (Leukemia inhibitory factor), an important factor in renal development, has been expressed by hAFSCs, inducing the proliferation rate of tubular epithelial cells [38].

Recently, the therapeutic effect of hAFSCs in acute kidney injury was also studied by Rota et al. [39]. The acute kidney injury murine model was generated by administrated the nephrotoxic drug, cisplatin, to NOD-SCID mice. c-Kit positive hAFSCs were intravenously injected 24 hours after injury. Limited tubular damage, improvement of the renal function and plolonged survival were observed. Transplantation of c-Kit positive hAFSCs was shown to inhibit apoptosis of tubular cells through the induction of the serinethreonine kinase Akt and in the same time to promote the proliferation of the renal tubular cells. These therapeutic effects were due to the paracrine action of the transplanted cKit positive hAFSCs that secreted factors such as IL-6, VEGF and SDF-1. In addition, this study suggested that hAFSCs pretreated with glial cell line derived neurotrophic factor (GDNF) enhanced their therapeutic effect by increasing the production of growth factors and the expression of receptors involved in cell homing and survival [39].

\section{Acute Hepatic Failure}

The description of acute hepatic failure involves the rapid loss of liver function, resulting in coagulopathy and hepatic encephalopathy. Recent research has been focused on the use of stem cell regenerative strategies, indicating an attractive alternative approach for liver repair.

In our recent studies, the effect of the transplantation of human SS-AF-MSCs or their conditioned medium was examined in acute hepatic failure [21]. NOD-SCID mice have been injected intraperitoneally with carbon tetrachloride $\left(\mathrm{CCL}_{4}\right)$ to generate the acute hepatic failure model. Twentyfour hours after the administration of $\mathrm{CCL}_{4}$, hAF-MSCs were transplanted in treated mice by intrahepatic or intraperitoneal injection. Interestingly, hAF-MSCs were engrafted into $\mathrm{CCL}_{4}$ injured livers, successfully downregulated the systemic inflammation and improved the liver function. We also observed a significant improvement in the liver function after intrahepatic delivery of conditioned medium from hAFMSCs, due to the cytokines and the growth factors that were secreted. Cytokines such as IL-10, IL-27, IL-17E, IL-12p70, IL-1 $\beta$ and IL-1ra, responsible for inducing local and systemic downregulation of pro-inflammatory mediators, were detected. This study clearly indicated the therapeutic effect of hAF-MSCs and point out the role of the paracrine effect and the secreted molecules from donor cells [21].

\section{Skin Wounds}

Injuries can become chronic wounds creating a significant burden for patients. Effective treatment of skin injuries requires full understanding of normal healing process. Recent studies provided evidence that fetal stem cells represent a promising tool for wound repair and tissue regeneration.

Yoon et al. generated a skin injury mouse model, creating two 2-mm full thickness excisional skin wounds on the side of the midline in each animal, in order to study the paracrine factors released by hAF-MSCs and their role in the wound healing process [40]. The conditioned medium of hAF-MSCs (hAF-MSC-CM) was subcutaneously injected around the wound and also topically applied in a donut shaped silicone splint on the wound. Using an antibody based protein array and ELISA method it was revealed that hAF-MSC-CM is comprised of cytokines and chemokines such as TNF- $\alpha$, VEGF, TGF- $\beta$, Leptin, IL6 and IL8 that play an important role in normal wound healing. In particular, hAF-MSC-CM through these secreted molecules induced the proliferation rate of dermal fibroblasts enhancing the wound healing process via TGF- $\beta /$ SMAD2 pathway. These data suggested that hAF-MSC-CM may represent a useful tool for tissue repair [40].

Recently, Mirabella et al. generated a fasciocutaneous flap model using Sprague Dawley rats [41]. Specifically, 
abdominal skin of rats was depilated and a square fasciocutaneous flap was elevated in a cranial to caudal direction as to be detached from the abdominal muscle layer. hAF-MSC$\mathrm{CM}$ was embedded to a gelatin membrane and then delivered on the muscular layer. Seven days after, the ischemic area was re-vascularized by the recruitment of endothelial progenitor cells as a result of the pro-angiogenic soluble factors secreted by hAF-MSCs. In particular, angiogenic growth factors and cytokines were secreted topically into the skin flap of the ischemic rat model, promoting the endogenous repair by recruiting endothelial progenitor cells [41].

\section{Ischemic Hind Limb}

Recently, it has been documented that hAFSC secretome was responsible for enhancing vasculogenesis and was capable of evoking a strong angiogenic response in an ischemic mouse model [42]. Ischemia was generated by unilateral ligation of the right femoral artery immediately distal to the origin of the deep femoral branch. c-Kit positive hAFSCs were embedded in two different biomaterials (polyurethane and matrigel) and implanted into the back of the mice. Twenty days after the transplantation, big blood vessels and collaterals were observed. In addition, the injured skin was repaired and vessel-perfused appeared. The conditioned medium was also added to liquid matrigel and then injected to the ischemic models. Improvement in the ischemia was observed through the promotion of neo-arteriogenesis. According to this study, hAFSC secretome was responsible for the stimulation of neo-arteriogenesis. Moreover, analysis of the conditioned medium derived from hAFSCs revealed the presence of known pro-angiogenic and anti-angiogenic factors, such as vascular endothelial growth factor (VEGF), stromal cell-derived factor-1 (SDF-1), interleukin 8 (IL-8), monocyte chemotactic protein-1 (MCP-1) and two angiogenesis inhibitors, interferon-gamma (IFN $\gamma$ ) and interferon gamma-induced protein 10 (IP-10) [42].

\section{Cancer}

Recent studies have proposed hAFSCs as cellular vehicles to deliver anti-cancer agents selectively to the tumor sites because of their homing properties in tumors [43].

An attempt to apply the stem cell based treatment in glioblastoma, the most malignant brain tumor, has been described recently from Yin et al. [44]. hAF-MSCs were stably transduced to deliver endostatin, an inhibitor of angiogenesis, and secreted carboxylesterase 2 (sCE2) that converts the prodrug CPT11 to the more active metabolite, SN-38. $\mathrm{BALB} / \mathrm{c}$ nude mice were transplanted with U87MG cells forming brain tumor and then hAF-MSC-endostatin-sCE2 were stereotactically injected into the left striata. Mice were also received CPT11 intravenously. Findings of this study suggested that hAF-MSC-endostatin-sCE2 cells remarkably decreased the vessel density, suppressed the cell proliferation rate and increased cell death in presence of CPT11. In parallel, a reduction of the nestin positive glioma stem cells and an increase in $\mathrm{GFAP}^{+}$and $\mathrm{S} 100 \beta^{+}$differentiated cells were reported [44].

Bitsika et al. introduced for the first time hAF-MSCs as useful tool for the treatment of a bladder tumor model [45].
Bladder cancer is a severe malignancy arising from the epithelial lining of the urinary bladder. In this study, immune-deficient mice were injected subcutaneously with T24M cells, an invasive and metastatic bladder cancer cell line and 10 days later tumors were established. hAF-MSCs were transduced with lentivirus carrying the sequence of human IFN $\beta$, resulting to the secretion of the IFN $\beta$ cytokine from the hAF-MSCs. These cells were administered in 3 weekly doses into the lateral tail vein of each animal and have been successfully migrated at the tumor site. Inhibition of the malignant tumor growth was detected, together with prolonged survival of mice in presence of IFN $\beta$-AF-MSCs. The mechanism proposed is based not only on the secretion of IFN $\beta$ but also on the paracrine effect generated by the produced cytokine [45].

A later study by Kang et al., investigated the therapeutic potential of hAFSCs in breast cancer [46]. Breast cancer is the most common invasive cancer in women and the treatment is based on surgery, chemotherapy and radiation with many side effects. A novel chemotherapy approach using genetically engineered hAFSCs to deliver suicide genes, such as cytosine deaminase (CD) and herpes simplex virus thymidine kinase (HSV-TK) was reported [46]. Suicide genes cause tumor cells death through apoptosis or programmed cell death, converting non-toxic prodrugs into toxic metabolites. In particular, $\mathrm{CD}$ gene converts prodrug 5fluorocytosine (5-FC) into a more toxic nucleotide analog, 5fluorouracil (5-FU), enhancing cell death through the formation of DNA double strand breaks [47]. Also, HSV-TK gene induces apoptosis, converting phosphorylate ganciclovir (GCV) into toxic metabolites [48]. hAFSCs were engineered to express these two suicide genes (AF.CD-TK) through a retroviral vector with $\mathrm{CD}-\mathrm{TK}$ fusion gene. Female BALB/c nude mice were injected subcutaneously with matrigel matrix contacting MDA-MB-231 cells into the right mammary fat pads. Mice developed tumors after 7 weeks and then were treated with the AF.CD-TK expressing cells in presence of $5-\mathrm{FU}$ and GCV prodrugs. It was reported that this treatment did not alter the structure of the breast tissue compare to the drug treatment. More importantly, AF.CD-TK expressing cells were detected at the tumor site and found to promote significantly the reduction of the tumor volume and weight without causing any undesirable side effects. Also, the presence of receptors such as c-Kit and CXCR4 in AF.CD-TK expressing cells, may facilitate their tropism to the tumor [46].

\section{Bladder Dysfunction in Parkinson Disease}

Parkinson disease is a degenerative disorder of the central nervous system. The progressive loss of dopamine generating cells in the substantia nigra leads to motor defects early in the course of the disease. Later, lower urinary symptoms may also be developed, resulting in a marked reduction in the quality of life Parkinson's patients. Dopamine agonists have been used in order to treat the early motor symptoms but these drugs eventually become ineffective. In these severe cases surgery and deep brain stimulation have been used, whereas currently active research directions are focused on gene therapy and stem cell transplants [49]. 
Table 1. Summary of hAFSC treatment into several disease animal models



Recent studies described the effect of cell therapy based on hAFSCs in bladder dysfunction using a well established rat model of Parkinson disease [50]. Soler et al. developed a dopaminergic lesion animal model in order to study the late phase of Parkinson disease, injecting the neurotoxin 6OHDA into the nigrostriatal pathway. Despite motor dysfunction, rats emerged bladder hyperactivity after the lesion. Transplantation of c-Kit positive hAFSCs significantly improved several urodynamic parameters but after 28 days the improvement recessed since cells were not detected, probably due to host immune reaction. More importantly, mice transplanted with hAFSCs revealed lower number of dead dopaminergic cells in the substantia nigra. It was proposed that hAFSCs after transplantation created a neuroprotective environment increasing the expression of various cytokines, such as IL-6, IL- $1 \alpha$ and IL-1 $\beta$. In addition, hAFSCs expressing the enzyme superoxide dismutase-2 (SOD-2) in vivo, contributed to the improvement of the oxidative stress created by the injection of 6-OHDA [50].

\section{CONCLUSIONS}

Cell based therapy is a promising strategy for treating numerous severe diseases. During the last years researchers have been focused on identifying the most appropriate stem cell type that can be used in such treatments providing remedial results. Extensive studies in several animal models suggested that hAFSCs may represent a valid alternative for regenerative medicine in the future. hAFSCs are thought to fit into an intermediate stage on the developmental continuum between embryonic and adult stem cells, mainly because of their fetal origin, their self-renewal capacity and their plasticity. Additionally, hAFSCs are easily accessible and exhibit minor or no ethical controversies. Another important characteristic is related to their immunological properties, reducing the risk of cell rejection and the potential of graft versus-host disease. More importantly, no evidence of tumorigenicity after hAFSC transplantation has yet been reported. The therapeutic effect observed by hAFSC transplantation clearly indicated that these cells are a effulgent source of well characterized immunomodulatory cells that exhibit extensive possibility for banking in the future and can be used for both autologous and allogeneic cell based therapy with promising remarkable results.

\section{CONFLICTS OF INTEREST}

The authors confirm that this article content has no conflicts of interest.

\section{ACKNOWLEDGEMENTS}

This study was supported by Grant «Heraklitos» No 70/3/11019 from the Greek Secretariat of Research and Technology that is co-financed by the European Social Fund and the Greek State. 


\section{REFERENCES}

[1] Daar AS, Greenwood HL. A proposed definition of regenerative medicine. J Tissue Eng Regen Med 2007; 1: 179-84.

[2] Hipp J, Atala A. Sources of stem cells for regenerative medicine. Stem Cell Rev 2008; 4: 3-11.

[3] Safinia L, Datan N, Hohse M, Mantalaris A, Bismarck A. Towards a methodology for the effective surface modification of porous polymer scaffolds. Biomaterials 2005; 26: 7537-47.

[4] Towns CR, Jones DG. Stem cells, embryos, and the environment: a context for both science and ethics. J Med Ethics 2004; 30: 410-3.

[5] Mason C, Brindley DA, Culme-Seymour EJ, Davie NL. Cell therapy industry: billion dollar global business with unlimited potential. Regen Med 2011; 6: 265-72.

[6] Prusa AR, Hengstschlager M. Amniotic fluid cells and human stem cell research: a new connection. Med Sci Monit 2002; 8: RA253-7.

[7] Roubelakis MG, Pappa KI, Bitsika V, et al. Molecular and proteomic characterization of human mesenchymal stem cells derived from amniotic fluid: comparison to bone marrow mesenchymal stem cells. Stem Cells Dev 2007; 16: 931-52.

[8] In 't Anker PS, Scherjon SA, Kleijburg-van der Keur C, et al. Amniotic fluid as a novel source of mesenchymal stem cells for therapeutic transplantation. Blood 2003; 102: 1548-9.

[9] Tsai MS, Lee JL, Chang YJ, Hwang SM. Isolation of human multipotent mesenchymal stem cells from second-trimester amniotic fluid using a novel two-stage culture protocol. Hum Reprod 2004; 19: $1450-6$.

[10] Tsai MS, Hwang SM, Tsai YL, et al. Clonal amniotic fluid-derived stem cells express characteristics of both mesenchymal and neural stem cells. Biol Reprod 2006; 74: 545-51.

[11] De Coppi P, Bartsch G, Jr., Siddiqui MM et al. Isolation of amniotic stem cell lines with potential for therapy. Nat Biotechnol 2007; 25: $100-6$.

[12] Roubelakis MG, Bitsika V, Zagoura $\mathrm{D}$, et al. In vitro and in vivo properties of distinct populations of amniotic fluid mesenchymal progenitor cells. J Cell Mol Med 2011; 15: 1896-913

[13] Leschot NJ, Verjaal M, Treffers PE. Risks of midtrimester amniocentesis; assessment in 3000 pregnancies. Br J Obstet Gynaecol 1985; 92: 804-7.

[14] Eddleman KA, Malone FD, Sullivan L, et al. Pregnancy loss rates after midtrimester amniocentesis. Obstet Gynecol 2006; 108: 106772.

[15] Delo DM, De Coppi P, Bartsch G, Jr., Atala A. Amniotic fluid and placental stem cells. Methods Enzymol 2006; 419: 426-38.

[16] Guillot PV, Gotherstrom C, Chan J, Kurata H, Fisk NM. Human first-trimester fetal MSC express pluripotency markers and grow faster and have longer telomeres than adult MSC. Stem Cells 2007; 25: 646-54.

[17] Prusa AR, Marton E, Rosner M, Bernaschek G, Hengstschlager M. Oct-4-expressing cells in human amniotic fluid: a new source for stem cell research? Hum Reprod 2003; 18: 1489-93.

[18] Kern S, Eichler H, Stoeve J, Kluter H, Bieback K. Comparative analysis of mesenchymal stem cells from bone marrow, umbilical cord blood, or adipose tissue. Stem Cells 2006; 24: 1294-301.

[19] D'Ippolito G, Schiller PC, Ricordi C, Roos BA, Howard GA. Agerelated osteogenic potential of mesenchymal stromal stem cells from human vertebral bone marrow. J Bone Miner Res 1999; 14: $1115-22$

[20] Moorefield EC, McKee EE, Solchaga L, et al. Cloned, CD117 selected human amniotic fluid stem cells are capable of modulating the immune response. PLoS One 2011; 6: e26535.

[21] Zagoura DS, Roubelakis MG, Bitsika V, et al. Therapeutic potential of a distinct population of human amniotic fluid mesenchymal stem cells and their secreted molecules in mice with acute hepatic failure. Gut 2012; 61: 894-906.

[22] Grisafi D, Piccoli M, Pozzobon M, et al. High transduction efficiency of human amniotic fluid stem cells mediated by adenovirus vectors. Stem Cells Dev 2008; 17: 953-62.

[23] Thomson JA, Itskovitz-Eldor J, Shapiro SS, et al. Embryonic stem cell lines derived from human blastocysts. Science 1998; 282: 1145-7.

[24] Zucchelli M, Strom S, Holm F, et al. In vivo differentiated human embryonic stem cells (hESC) can acquire more frequently chromosomal aberrations than in vitro during the same period. Stem Cells Dev 2012
Prokhorova TA, Harkness LM, Frandsen U, et al. Teratoma formation by human embryonic stem cells is site dependent and enhanced by the presence of Matrigel. Stem Cells Dev 2009; 18: 47-54.

[26] Steindler DA. Stem cells, regenerative medicine, and animal models of disease. Ilar J 2007; 48: 323-38.

[27] Zhao P, Ise H, Hongo M, et al. Human amniotic mesenchymal cells have some characteristics of cardiomyocytes. Transplantation 2005; 79: 528-35.

[28] Chiavegato A, Bollini S, Pozzobon M, et al. Human amniotic fluidderived stem cells are rejected after transplantation in the myocardium of normal, ischemic, immuno-suppressed or immunodeficient rat. J Mol Cell Cardiol 2007; 42: 746-59.

[29] Delo DM, Guan X, Wang Z, et al. Calcification after myocardial infarction is independent of amniotic fluid stem cell injection. Cardiovasc Pathol 2011; 20: e69-78.

[30] Cipriani S, Bonini D, Marchina E, et al. Mesenchymal cells from human amniotic fluid survive and migrate after transplantation into adult rat brain. Cell Biol Int 2007; 31: 845-50.

[31] Pan HC, Chen CJ, Cheng FC, et al. Combination of G-CSF administration and human amniotic fluid mesenchymal stem cell transplantation promotes peripheral nerve regeneration. Neurochem Res 2009; 34: 518-27.

[32] Pan HC, Chin CS, Yang DY, et al. Human amniotic fluid mesenchymal stem cells in combination with hyperbaric oxygen augment peripheral nerve regeneration. Neurochem Res 2009; 34: 1304-16.

[33] Pan HC, Yang DY, Ho SP, et al. Escalated regeneration in sciatic nerve crush injury by the combined therapy of human amniotic fluid mesenchymal stem cells and fermented soybean extracts, Natto. J Biomed Sci 2009; 16: 75.

[34] Cheng FC, Tai MH, Sheu ML, et al. Enhancement of regeneration with glia cell line-derived neurotrophic factor-transduced human amniotic fluid mesenchymal stem cells after sciatic nerve crush injury. J Neurosurg 2010; 112: 868-79.

[35] Yang DY, Sheu ML, Su HL, et al. Dual regeneration of muscle and nerve by intravenous administration of human amniotic fluidderived mesenchymal stem cells regulated by stromal cell-derived factor-1alpha in a sciatic nerve injury model. J Neurosurg 2012; 116: 1357-67.

[36] Perin L, Giuliani S, Jin D, et al. Renal differentiation of amniotic fluid stem cells. Cell Prolif 2007; 40: 936-48.

[37] Perin L, Sedrakyan S, Giuliani S, et al. Protective effect of human amniotic fluid stem cells in an immunodeficient mouse model of acute tubular necrosis. PLoS One 2010; 5: e9357.

[38] Hauser PV, De Fazio R, Bruno S, et al. Stem cells derived from human amniotic fluid contribute to acute kidney injury recovery. Am J Pathol 2010; 177: 2011-21.

[39] Rota C, Imberti B, Pozzobon M et al. Human amniotic fluid stem cell preconditioning improves their regenerative potential. Stem Cells Dev 2012; 21: 1911-23.

[40] Yoon BS, Moon JH, Jun EK et al. Secretory profiles and wound healing effects of human amniotic fluid-derived mesenchymal stem cells. Stem Cells Dev 2010; 19: 887-902.

[41] Mirabella T, Hartinger J, Lorandi C, et al. Proangiogenic Soluble Factors from Amniotic Fluid Stem Cells Mediate the Recruitment of Endothelial Progenitors in a Model of Ischemic Fasciocutaneous Flap. Stem Cells Dev 2012; 21: 2179-88

[42] Mirabella T, Cilli M, Carlone S, Cancedda R, Gentili C. Amniotic liquid derived stem cells as reservoir of secreted angiogenic factors capable of stimulating neo-arteriogenesis in an ischemic model. Biomaterials 2011; 32: 3689-99.

[43] Sasportas LS, Kasmieh R, Wakimoto H, et al. Assessment of therapeutic efficacy and fate of engineered human mesenchymal stem cells for cancer therapy. Proc Natl Acad Sci USA 2009; 106: 4822-7.

[44] Yin J, Kim JK, Moon JH, et al. hMSC-mediated concurrent delivery of endostatin and carboxylesterase to mouse xenografts suppresses glioma initiation and recurrence. Mol Ther 2011; 19: 11619.

[45] Bitsika V, Roubelakis MG, Zagoura D, et al. Human amniotic fluid-derived mesenchymal stem cells as therapeutic vehicles: a novel approach for the treatment of bladder cancer. Stem Cells Dev 2012; 21: 1097-111.

[46] Kang NH, Hwang KA, Yi BR, et al. Human amniotic fluid-derived stem cells expressing cytosine deaminase and thymidine kinase in- 
hibits the growth of breast cancer cells in cellular and xenograft mouse models. Cancer Gene Ther 2012; 19: 412-9.

[47] Myers CE. The pharmacology of the fluoropyrimidines. Pharmacol Rev 1981; 33: 1-15.

[48] Tomicic MT, Thust R, Kaina B. Ganciclovir-induced apoptosis in HSV-1 thymidine kinase expressing cells: critical role of DNA breaks, Bcl-2 decline and caspase-9 activation. Oncogene 2002; 21: 2141-53.
[49] Obeso JA, Rodriguez-Oroz MC, Goetz CG, et al. Missing pieces in the Parkinson's disease puzzle. Nat Med 2010; 16: 653-61.

[50] Soler R, Fullhase C, Hanson A, et al. Stem cell therapy ameliorates bladder dysfunction in an animal model of Parkinson disease. J Urol 2012; 187: 1491-7. 